\title{
Surgical aspects, violations and outcomes of Wilms tumor-a multicenter study in a resource-limited country
}

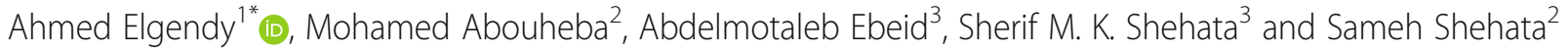

\begin{abstract}
Background: Wilms tumor is the commonest malignant renal neoplasm in children. Surgery plays a pivotal role in the management, and evidence-based guidelines for surgical resection have been established by the major international groups. Any deviation from the protocol is considered as a violation. The goal of this study was to evaluate outcomes of the patients with unilateral Wilms tumor treated at a developing country and to analyze surgical violations (SV) and their impact on the prognosis. A retrospective review was conducted for 37 patients who were presented to our hospitals and underwent nephrectomy for WT from January 2016 to December 2018. All participating centers adopt Children's Oncology Group protocol. The SV were analyzed by logistic regression. Overall survival (OS) and event-free survival (EFS) were estimated by the Kaplan-Meier method.

Results: There were 12 (32.4\%), 11 (29.7\%), 10 (27\%), and 4 (10.8\%) stages I, II, III, and IV, respectively. Their median age at time of diagnosis was 3.1 years. Upfront nephrectomy was performed for 30 cases. Six patients had tumor relapse (2 lungs and 4 local recurrences) at a median follow-up of 15.7 months. Out of the relapsed patients, two had unfavorable histology, and regarding their staging, four were stage III, one was stage II, and one was stage IV. Thirty-month OS and EFS were $84.3 \%$ and $81.1 \%$, respectively. Twenty-seven SV occurred within 25 patients. Lack or inadequate lymph node sampling represented $74.07 \%$ (20/27), intraoperative tumor rupture and spillage accounted for $18.52 \%(5 / 27)$, and unwarranted preoperative biopsy happened in $7.41 \%(2 / 27)$. The SV were not correlated with mortality ( $p$ value $=0.381$ ); however, they had a significant impact on the relapse $(p$ value $=0.001)$. On further analysis; tumor rupture and spillage was a predictor for recurrence reaching a statistical significance $(p$ value $=0.003)$, whereas the other violations were not.

Conclusions: Favorable outcomes could be achieved by compliance with evidence-based guidelines even in a resource-limited country like ours. Violations were correlated with relapse; however, only tumor rupture and spillage was of statistical significance in multivariate analysis. Failure of lymph node documentation was the main problem encountered, and it should be avoidable in future practice.
\end{abstract}

Keywords: Wilms tumor, Nephrectomy, Surgical violations, Resource-limited country

\footnotetext{
* Correspondence: ahmed.elgendy@med.tanta.edu.eg

This study has been presented as an Oral Presentation by the Corresponding Author at the $20^{\text {th }}$ European Pediatric Surgical Association Congress (EUPSA 2019), Belgrade, Serbia.

'Surgical Oncology Unit, Tanta University, 35 Ali Beek Elkbeer street, Tanta 31515, Egypt

Full list of author information is available at the end of the article
}

\section{Springer Open}

(c) The Author(s). 2020 Open Access This article is licensed under a Creative Commons Attribution 4.0 International License, which permits use, sharing, adaptation, distribution and reproduction in any medium or format, as long as you give appropriate credit to the original author(s) and the source, provide a link to the Creative Commons licence, and indicate if changes were made. The images or other third party material in this article are included in the article's Creative Commons licence, unless indicated otherwise in a credit line to the material. If material is not included in the article's Creative Commons licence and your intended use is not permitted by statutory regulation or exceeds the permitted use, you will need to obtain permission directly from the copyright holder. To view a copy of this licence, visit http://creativecommons.org/licenses/by/4.0/. 


\section{Background}

Wilms tumor [WT] is the commonest malignant renal neoplasm in children, and it is also considered as the third most frequently encountered malignancy in the pediatric age group [1]. The management of WT is a true story of success regarding the multimodal treatment in children's oncology. Recent reports indicate that about $90 \%$ of the patients suffering from this tumor could have an opportunity to be cured [2].

Surgery always plays a pivotal role in any therapeutic strategy directed towards this tumor, and definitely, complete tumor resection is the cornerstone of the management and prognosis. The evidence-based guidelines for surgical resection of WT have been established and recommended by the major international groups' studies [3-5]. Following precise surgical guidelines is fundamental for obtaining favorable results and any deviation from the protocol is considered as a violation [6].

The treatment of WT in health facilities with limited resources represents a major challenge facing pediatric oncology surgeons [7]. The goal of this study was to evaluate short-term clinical outcomes of the patients with unilateral WT in a multicenter study conducted at a developing country. Another purpose was to analyze surgical aspects performed during nephrectomies, types and numbers of iatrogenic protocol violations, and their impact on the prognosis.

\section{Methods}

This is a retrospective study of prospectively collected data including all children with unilateral WT who were managed at three surgical units and their affiliated regional centers by five consultant surgeons between January 2016 and December 2018. Patients with bilateral tumors were excluded from the analysis. Following obtaining IRB approval from all participating centers, data were retrieved and collated in a single sheet for reviewing patients' characteristics, radiological findings, treatment regimens, full surgical details, postoperative pathological reports, complications, survival data, and outcome. Informed consents were signed by all parents for surgery, anesthesia and data use in scientific purposes only.

The management protocol in all participating units was uniform and adopted accordingly to Children's Oncology Group [COG] guidelines, formerly named $\mathrm{Na}$ tional Wilms Tumor Study Group [NWTSG] [8, 9]. Patients were diagnosed by abdominal ultrasound (US) and computed tomography (CT). US was used for confirmation of the renal origin of the tumor, and the assessment of tumor extension and lymph node status were based on CT. Chest X-ray and CT were performed for the detection of pulmonary metastases. Tumor staging, histopathological typing, and adjuvant therapy were based on the same aforementioned protocol. Patients were closely monitored till January 2019, and follow-up visits were scheduled at the outpatients' clinics or by contact with the parents via telephone.

The violations of the current protocol were analyzed regarding the types, rates, and their impact on the outcome. They included unwarranted or unnecessary preoperative biopsy which causes tumor upstaging, incorrect abdominal incisions, intraoperative tumor rupture and spillage, absence or inadequate lymph node sampling [less than 7 nodes], and extensive resection of vital organs [other than the adrenal gland or a small part of the diaphragm] as reported in former literature reports $[4,10]$. Figure 1 shows current COG recommendations regarding the timing of management for stage III and IV tumors.

Statistical data analysis was performed using SPSS (Statistical Package for Social Science version 21.0). Overall survival (OS) and event-free survival (EFS) rates were estimated using the Kaplan-Meier method and reported at 30 months. OS was calculated from the date of diagnosis to the date of death or last follow-up, while EFS was measured from the date of diagnosis to the date of any event. Surgical violations were analyzed by logistic regression. A $p$ value equal or less than 0.05 was considered to be of statistical significance.

\section{Results}

\section{Patients' characteristics}

A total of 37 patients with full details were included and analyzed. There were 21 males and 16 females. Median age at diagnosis was 3.1 years (range $0.33-8.5$ years). Palpable abdominal mass was the most common presentation among children in this study (20 cases). Twenty patients were presented with left-sided tumors, while the remaining 17 had right-sided lesions. Out of all cases,

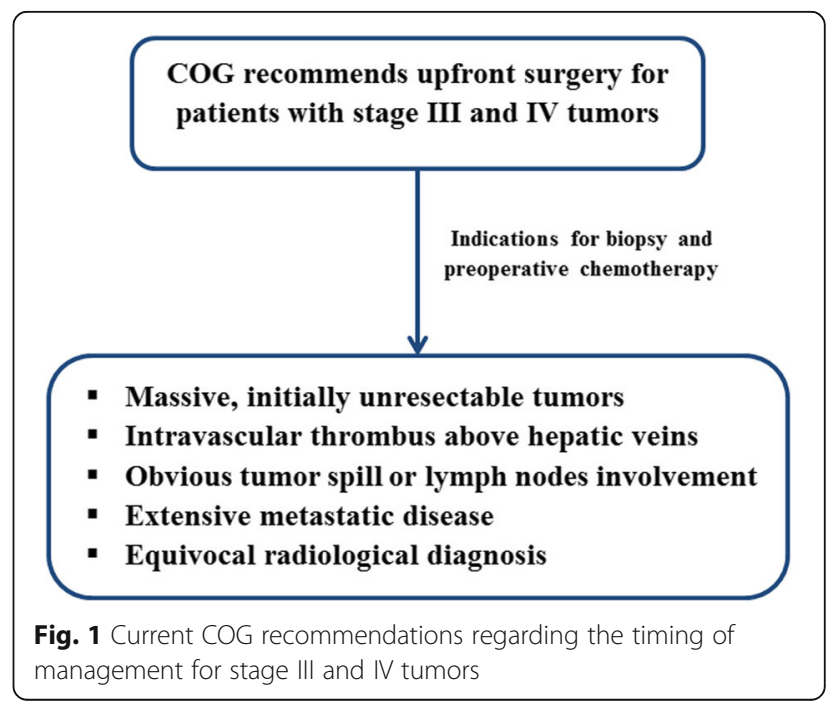


Table 1 The patients' demographics, clinical characteristics, and staging

\begin{tabular}{|c|c|}
\hline Parameters & $N(\%)$ \\
\hline \multicolumn{2}{|l|}{ Gender } \\
\hline Male & $21(56.8 \%)$ \\
\hline Female & $16(43.2 \%)$ \\
\hline \multicolumn{2}{|l|}{ Age } \\
\hline Under 3 years & $20(54.1 \%)$ \\
\hline Above 3 years & $17(45.9 \%)$ \\
\hline \multicolumn{2}{|l|}{ Main mode of presentation } \\
\hline Palpable abdominal mass & $20(54.1 \%)$ \\
\hline Increased abdominal girth and distention & $6(16.2 \%)$ \\
\hline Abdominal pain & $5(13.5 \%)$ \\
\hline Hematuria & $3(8.1 \%)$ \\
\hline Recurrent fever & $3(8.1 \%)$ \\
\hline \multicolumn{2}{|l|}{ Metastases at diagnosis } \\
\hline Metastatic (lung) & $4(10.8 \%)$ \\
\hline Non-metastatic & $33(89.2 \%)$ \\
\hline \multicolumn{2}{|l|}{ Tumor side } \\
\hline Left & $20(54.1 \%)$ \\
\hline Right & $17(45.9 \%)$ \\
\hline \multicolumn{2}{|l|}{ Associated anomalies } \\
\hline No & $32(86.5 \%)$ \\
\hline Inguinal hernia & $3(8.1 \%)$ \\
\hline Undescended testicles & $1(2.7 \%)$ \\
\hline Hypospadias & $1(2.7 \%)$ \\
\hline \multicolumn{2}{|l|}{ Staging according to COG } \\
\hline । & $12(32.4 \%)$ \\
\hline$\|$ & $11(29.7 \%)$ \\
\hline III & $10(27 \%)$ \\
\hline IV & $4(10.8 \%)$ \\
\hline \multicolumn{2}{|l|}{ Histopathology } \\
\hline Favorable & $33(89.2 \%)$ \\
\hline Unfavorable & $4(10.8 \%)$ \\
\hline
\end{tabular}

four had lung metastases at time of diagnosis and five had associated non-syndromic anomalies. Follow-up time ranged between 1.4 and 35.5 months with a median of 15.7 months. The patients' demographics, clinical characteristics, and staging are summarized in Table 1.

\section{Management and complications}

Primary surgery was planned to be performed for all cases as shown in Figs. 2 and 3; however, thirty patients had upfront nephrectomy. The remaining 7 children underwent post-chemo resection after an imagingguided tru-cut biopsy. Four of them were metastatic and the other three were stage III. Transverse transperitoneal

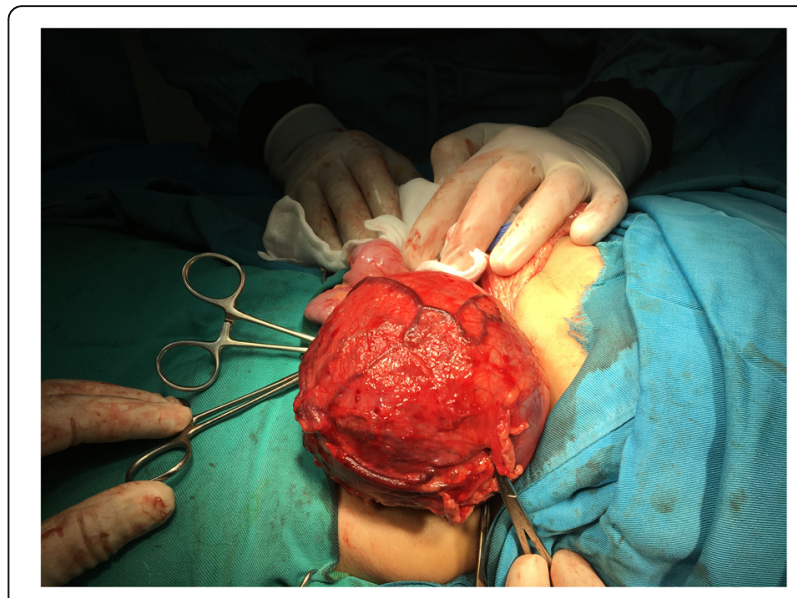

Fig. 2 Upfront nephrectomy

incision was adopted in all nephrectomies without reporting of any violations. There were 12 (32.4\%), 11 (29.7\%), $10(27 \%)$, and $4(10.8 \%)$ patients with WT classified into stages I, II, III, and IV, respectively as per the current COG protocol [9]. Four patients had unfavorable histology (one was stage III and 3 were stage IV). Out of all patients, 7 had different postoperative staging with regard to initial staging, tumor rupture occurred in 5 patients, and 2 had positive lymph nodes metastases.

Nineteen patients $(51.35 \%)$ had adrenalectomy as a part of en bloc resection during nephrectomy. Two of them had additional resection of a small part of the diaphragm due to tumor adherence. Five patients (13.5\%) had tumor rupture and spillage intraoperatively (3 left and 2 right-sided) during attempts of primary surgery as shown in Figs. 4 and 5. There were no other intraoperative complications or mortality. Regional lymph node sampling from the renal bed and around major vessels was performed in 29 patients (78.4\%) with a median of 7 nodes (range 2-11); 12 of them had less than 7 nodes

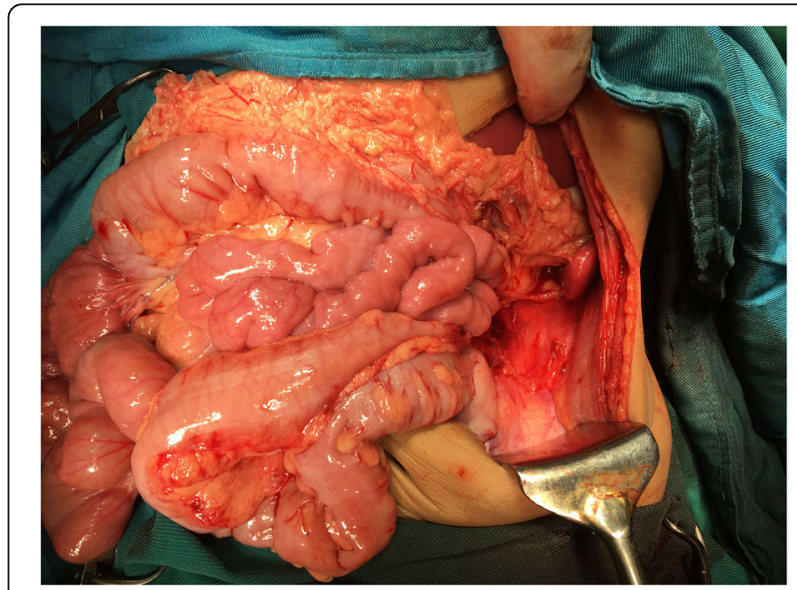

Fig. 3 Bloodless operative field after primary surgery 


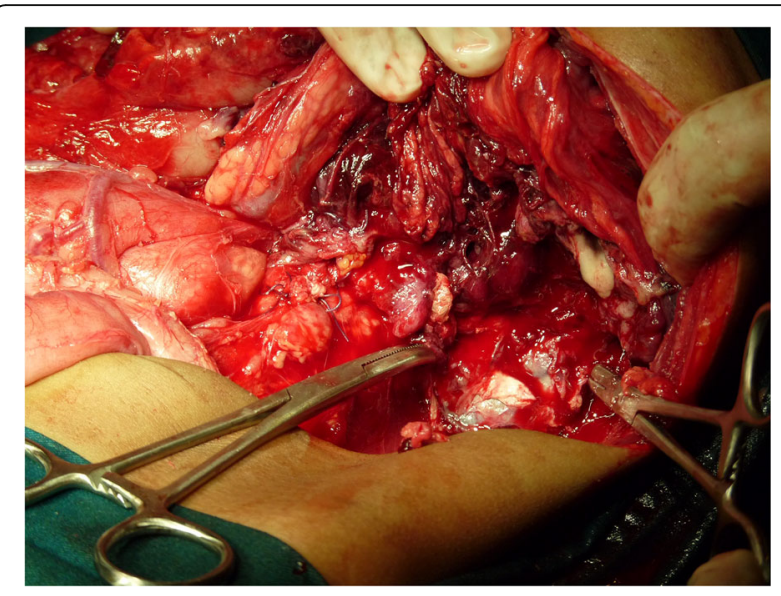

Fig. 4 Intraoperative tumor rupture and spillage during upfront surgery

sampled. While in the remaining 8 , no lymph nodes were retrieved from the specimen. Out of all patients; three (8.1\%) had postoperative complications, one developed intussusception on the 5th day after surgery that was managed by laparotomy and reduction and two patients readmitted with adhesive intestinal obstruction during receiving adjuvant therapy, and both of them underwent small intestinal resection anastomosis.

Regarding additional therapies, all patients received adjuvant chemotherapy and 14 had post-excision radiotherapy (10 cases stage III and 4 cases stage IV). Flank radiation was given to 9 patients, whereas the other 5 received whole abdomen radiotherapy due to the occurrence of tumor rupture and spillage.

\section{Outcome and survival analysis}

Six patients had tumor relapse; two of them had lung recurrence. The other four had local recurrence (2 at the

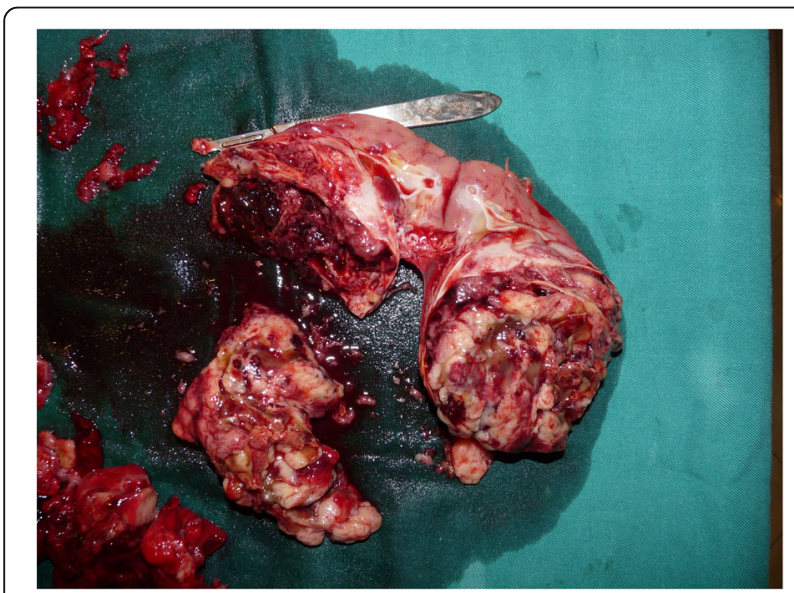

Fig. 5 Tumor rupture and spillage during upfront surgery operative bed, one at the para-aortic area, and one at segment VI of the liver). All locally relapsed patients had tumor rupture and spillage during primary surgery, and no lymph nodes were sampled in one of them. All cases with recurrences received intensive chemotherapy and two patients underwent secondary surgery (one for hepatic recurrence and the other for tumor bed relapse). Disease progression occurred in three children (2 with lung recurrence and one with local relapse) and died without any surgical intervention. Their histology was as follows: 2 with lung relapse (one favorable and unfavorable) and one with local recurrence (favorable histology). At the end of follow-up, thirty-four patients were still alive. Thirty-month OS and EFS for all patients were $84.3 \%$ and $81.1 \%$, respectively (Figs. 6 and 7).

\section{Surgical protocol's violations}

Twenty-seven violations occurred within 25 patients. Failure of lymph node documentation either absence or inadequate sampling (less than 7 nodes) were encountered in 18 patients. Intraoperative tumor rupture and spillage occurred in three, two patients had a double violation (inadequate sampling in addition to tumor spillage), and 2 cases had unwarranted preoperative tumor biopsy. The types and rates of surgical violations practiced in this study are listed in Table 2.

Absence or inadequate lymph node documentation was the most common problem representing about $74 \%$ $(20 / 27)$ of all violations. In 8 of them, lymph nodes were not sampled at all. Whereas in the remaining 12 , the retrieved nodes were less than 7 nodes (range 2-4) and all of them were negative for malignancy. Fourteen cases were of stages I and II among the patients who had lymph node violations. Regarding the 7 children who underwent imaging-guided preoperative biopsy, 5 had biopsy due to a doubtful radiological diagnosis with neuroblastoma (not a protocol violation), while 2 patients had an unwarranted biopsy, and this is considered as a violation according to the current COG guidelines. Patients staged as stage III were due to intraoperative tumor rupture in $50 \%(5 / 10$, one of them also had positive nodes), while preoperative tumor biopsy was responsible for $30 \%(3 / 10,2$ of them also had positive nodes) and the remaining 20\% (2/10) were due to positive malignant lymph nodes. Figure 8 demonstrates the occurrence of violations in relation to tumor stage.

Regarding the influence of surgical violations on the survival outcome, they were not correlated with mortality ( $p$ value $=0.381)$; however, they had a significant impact on tumor relapse ( $p$ value $=0.001)$. On multivariate analysis, intraoperative tumor rupture and spillage was reported as the only significant predictor for recurrence among all violations $(p$ value $=0.003)$. The logistic 


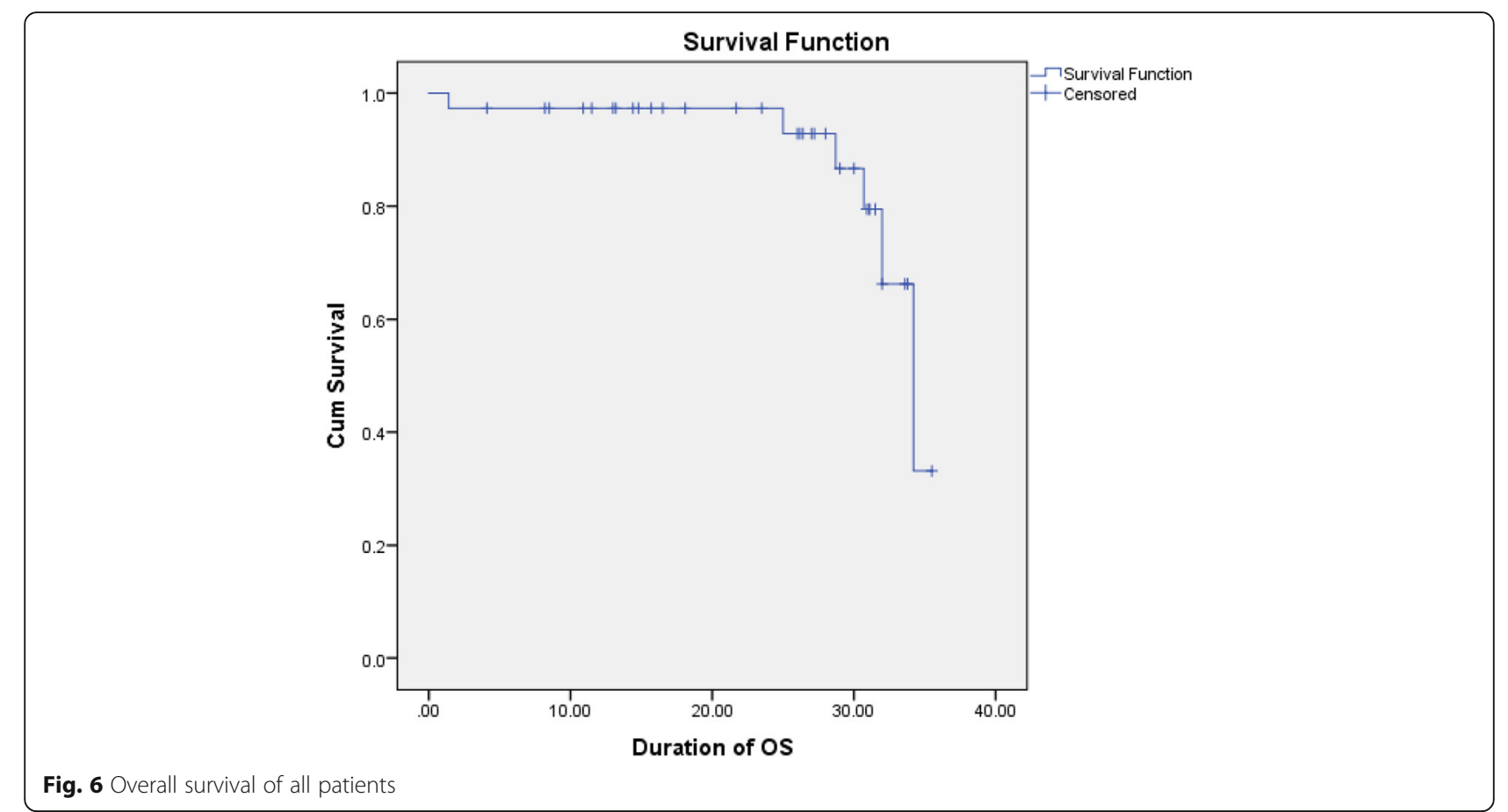

regression of surgical violations on the relapse is shown in Table 3.

\section{Discussion}

Management of WT in developing countries provides well-known challenges such as late presentations particularly in malnourished children, failure or abandonment of therapy, insufficient capacity of specialized hospitals, and deficiency in treatment facilities [11, 12]. Some experts analyzed that primary surgery might be unsuitable to be practiced in resource-constrained settings due to the aforementioned problems; therefore, delayed resection should be the principal modality [13]. The current study has special considerations as being carried out in a resource-limited society where patients presented with huge masses due to delayed diagnosis;

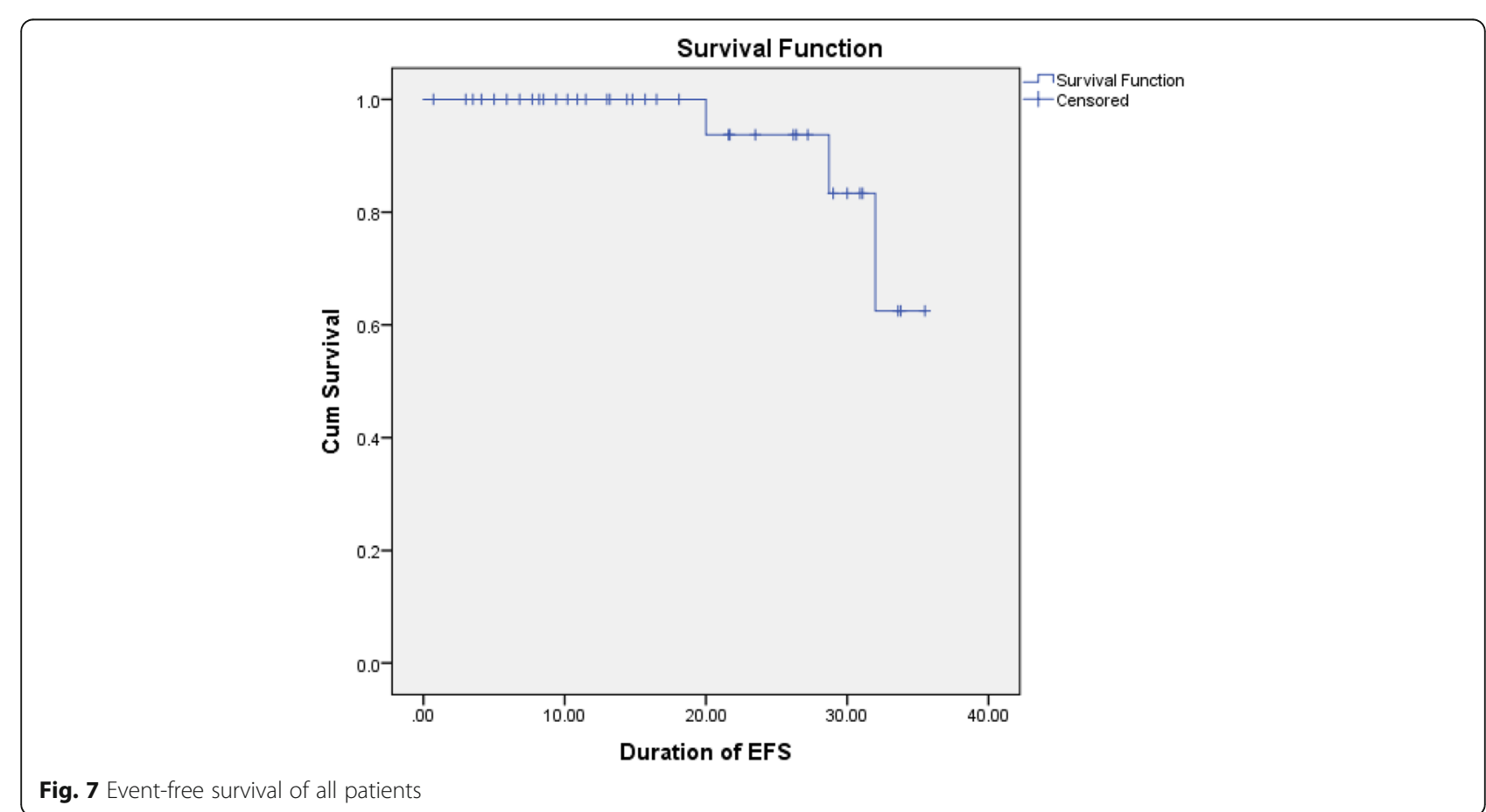


Table 2 The types and rates of surgical violations practiced in this study

\begin{tabular}{ll}
\hline Type of violation & $N(\%)$ \\
\hline Failure of lymph nodes documentation & $20(74.07 \%)$ \\
Intraoperative tumor rupture and spillage & $5(18.52 \%)$ \\
Unnecessary or unwarranted preoperative tumor biopsy & $2(7.41 \%)$
\end{tabular}

meanwhile, surgeons adopt upfront nephrectomy and COG guidelines.

Median age at presentation in this study was found to be nearly the same compared to other reports from North Africa, Asia, and Europe [7, 14, 15], and slightly lower than that reported by a North American study [16]. Gender distribution among our cases was in favor of males and the same result was documented in an Asian study [14], in contrast with Western data [15]. Palpable abdominal mass was the commonest presentation in this study and the same findings were observed in other studies conducted at developing countries [7, 14], while other complaints in addition to palpable mass were reported in African patients due to a more advanced disease [17]. In contrast, there were earlier referrals in affluent societies, and even there were differences between them regarding the percentage of cases discovered incidentally. PritchardJones et al. observed that a lesser proportion of patients in the UK were diagnosed incidentally when compared to Germany [18].

The percentage of metastatic disease was $10.8 \%$ in this study, and this was similar to an Indian study [19]. Interestingly, this incidence was lesser when compared to several studies at other developing countries ranging between 14 and $30.5 \%$ including one of them also reported in India [14, 20-22]. Such difference could be due to the number of patients, duration of series, and referral bias and might be that locally advanced tumors were more than the metastatic disease as in our cohort.
The majority of patients in this study were managed by primary surgery. Although the aforementioned difficulties of selecting such protocol in our environment, it was positive to observe that intraoperative complications were only due to tumor ruptures, and there were no other operative morbidity or mortality. However, relapse related to rupture was a cause of postoperative death in one patient. It is also very interesting to notice that some centers in developing countries adopt the International Society of Paediatric Oncology (SIOP) protocol [7, 14, 22] in order to overcome the delayed presentations, whereas others depend on COG recommendations [20, 23, 24].

The participating centers in this study prefer COG guidelines to avoid administration of preoperative chemotherapy to non-WT or benign disease [13]. Additionally, there is an evident psychological factor of upfront nephrectomy on families in our community and their comfort towards surgery as a primary step. Relying on the SIOP protocol needs specialized radiologists and pathologists to avoid imaging misdiagnosis or understaging due to change of tumor histology, whereas COG protocol exposes patients to more abdominal radiation due to more tumor rupture [13]. Eventually, the end results are very similar between both approaches [25], and every center whatever its location can choose according to the experiences.

The survival rates were reported above 90\% in highincome countries [21]. In this study, such data was better than the declared in a previous national series [OS 78.9\%] [26]. Meanwhile, our results were within reasonable range among those reported from other developing countries in North Africa and Asia [OS 74-89\%, EFS 73-86\%] [7, 14, 19, 20]. Impressive outcomes were achieved by a study in Latin America with OS and EFS of $91 \%$ and $85 \%$, respectively [22]. While, dismal survival rates were observed in sub-Saharan Africa of 25-46\% $[17,21]$.

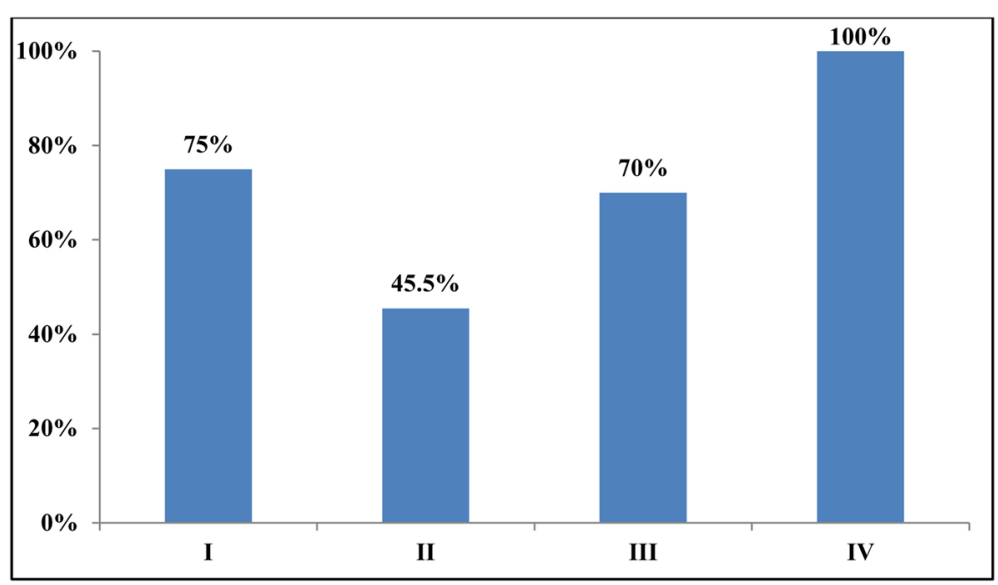

Fig. 8 The occurrence of violations in relation to tumor stage 
Table 3 The logistic regression of types of surgical violations on relapse

\begin{tabular}{lll}
\hline Type of violation & HR $(95 \% \mathrm{Cl})$ & $p$ value \\
\hline Failure of lymph nodes documentation & $1.380(0.109-17.343)$ & 0.803 \\
Tumor rupture and spillage & $6.820(3.929-9.461)$ & $0.003^{*}$ \\
Unnecessary or unwarranted preoperative biopsy & $4.879(0.248-8.571)$ & 0.857 \\
\hline
\end{tabular}

*Significant

Improvements in the management could be achieved by adherence to the evidence-based guidelines and avoidance of violations. Due to the crucial role of surgeons in complete resection, staging, and avoiding tumor rupture, recommended surgical guidelines must be adopted in order to optimize outcomes [4]. Despite the high percentage of violations practied in this study, the majority of them lack or fault in lymph node management, and this was similar when compared to a recent COG study reporting that $65 \%$ of all violations were absence of lymph node sampling [6]. Furthermore, the same problem was declared in an SIOP study, which documented that incorrect sampling occurred in $88.2 \%$ of patients [27]. The minimum of seven nodes sampled is crucial for detecting metastases by COG [6], and it is of six nodes by SIOP, which formerly reported that only three nodes were sufficient $[27,28]$. Such violation remains the commonest mistake made by surgeons everywhere, and its consequences lead to less aggressive adjuvant therapy and high risk of recurrence. However, it was not correlated with relapse in our series and also had no impact on EFS by a COG report [29].

Tumor rupture was documented as a predictor for recurrence as per NWTSG [5], and we observed the same with a significant difference in this study. Intraoperative tumor rupture in this cohort was similar to that reported by a COG report (11.5\%) [30], whereas it was higher than the declared by an SIOP study (1.45\%) [27]. This notable difference is due to adoption of delayed surgery by SIOP and the role of preoperative chemotherapy in making tumors more solid and downsized. Biopsy due to an equivocal initial diagnosis with other neoplasms, such as nueroblastoma or lymphoma, was not considered as a violation in the current protocol [6]. Thus, this study had minor violations of unwarranted preoperative biopsy. We also did not report any violation regarding surgical incisions or extensive organs resection. Finally, The authors of this study propose that careful assessment of resectability in multidisciplinary team meetings and adequate lymph node sampling can surely reduce the occurrence of violations in the future.

Several limitations were observed in this study as its retrospective nature, small sample size, and few numbers of participating centers with short-term results. The last drawback might be due to lacking of registration systems in our nation and the authors invited other centers to share their experience; however, data loss was the main obstacle. We believe that a further study with more number of patients is warranted for more confirmation.

\section{Conclusions}

Favorable outcomes could be achieved by compliance with evidence-based guidelines even in a resourcelimited country like ours. Surgical violations were correlated with relapse; however, multivariate analysis showed that only tumor rupture and spillage was of statistical significance. Failure of lymph node documentation was the main problem encountered, and it should be avoidable in future practice.

\section{Abbreviations \\ WT: Wilms tumor; COG: Children's Oncology Group; NWTSG: National Wilms Tumor Study Group; SV: Surgical violations; OS: Overall survival; EFS: Event- free survival; SIOP: International Society of Paediatric Oncology; US: Ultrasound; CT: Computed tomography}

\section{Acknowledgements}

The authors would like to thank Mr. Ahmed Samir Ryad for the technical assistance in the manuscript.

\section{Authors' contributions}

Study design: A E. Data collection: A E, M A, and Ab E. Manuscript writing: A E. Manuscript revision: A E, S M K S, and S S. All authors read and approved the final manuscript.

\section{Funding}

Not applicable.

\section{Availability of data and materials}

The datasets used and/or analyzed during the current study are available from the corresponding author on reasonable request.

\section{Ethics approval and consent to participate}

This is a retrospective review and is neither prospective nor experimental. A research ethical approval was not applicable; however, approval for the data collection and analysis for the purpose of the study was obtained from the research and scientific committee of the Surgical Oncology Unit and Pediatric Surgery Department at Tanta University, and Pediatric Surgery Department at Alexandria University in January 2019.

\section{Consent for publication}

Informed consents were signed by all parents for surgery and data use in scientific purposes only at the time of management at Tanta University and Alexandria University. This consent was clearly stated in the manuscript in the "Methods" section. All the private data of patients such as name, address, and phone number or even identity photos will not appear in the research.

The consents are in the patients' medical records.

Competing interests

The authors declare that they have no competing interests. 


\section{Author details}

'Surgical Oncology Unit, Tanta University, 35 Ali Beek Elkbeer street, Tanta 31515, Egypt. ${ }^{2}$ Pediatric Surgery Department, Alexandria University, Alexandria, Egypt. ${ }^{3}$ Pediatric Surgery Department, Tanta University, Tanta, Egypt.

Received: 16 January 2020 Accepted: 3 June 2020

Published online: 29 June 2020

\section{References}

1. Millar AJW, Cox S, Davidson A (2017) Management of bilateral Wilms tumours. Pediatr Surg Int 33(7):737-745

2. Godzinski J, Graf N, Audry G (2014) Current concepts in surgery for Wilms tumor--the risk and function-adapted strategy. Eur J Pediatr Surg 24(6):457-460

3. Fuchs J, Kienecker K, Furtwangler R, Warmann SW, Burger D, Thurhoff JW et al (2009) Surgical aspects in the treatment of patients with unilateral Wilms tumor. A report from the SIOP 93-01/German Society of Pediatric Oncology and Hematology. Ann Surg 294(4):666-671

4. Ehrlich PF, Ritchey ML, Hamilton TE, Haase GM, Ou S, Breslow N et al (2005) Quality assessment for Wilms' tumor: a report from the National Wilms' Tumor Study-5. J Pediatr Surg 40(1):208-212

5. Shamberger RC, Guthrie KA, Ritchey ML, Haase GM, Takashima J, Beckwith JB et al (1999) Surgery-related factors and local recurrence of Wilms tumor in National Wilms Tumor Study 4. Ann Surg 229(2):292-297

6. Ehrlich PF, Hamilton TE, Gow K, Barnhart D, Ferrer F, Kandel J et al (2016) Surgical protocol violations in children with renal tumors provides an opportunity to improve pediatric cancer care: a report from the Children's Oncology Group. Pediatr Blood Cancer 63(11):1905-1910

7. Madani A, Zafad S, Harif M, Yaakoubi M, Zamiati S, Sahraoui S et al (2006) Treatment of Wilms tumor according to SIOP 9 protocol in Casablanca, Morocco. Pediatr Blood Cancer 46(4):472-475

8. Kalapurakal JA, Dome JS, Perlman EJ, Malogolowkin M, Haase GM, Grundy P et al (2004) Management of Wilms' tumour: current practice and future goals. Lancet Oncol 5(1):37-46

9. Irtan S, Ehrlich PF, Pritchard-Jones K (2016) Wilms tumor: "state-of-the-art" update. Semin Pediatr Surg 25(5):250-256

10. Kieran K, Ehrlich PF (2016) Current surgical standards of care in Wilms tumor. Urol Oncol 34(1):13-23

11. Hadley LG, Rouma BS, Saad-Eldin Y (2012) Challenge of pediatric oncology in Africa. Semin Pediatr Surg 21(2):136-141

12. Israels T, Moreira C, Scanlan T, Molyneux L, Kampondeni $S$, Hesseling $P$ et al (2013) SIOP PODC: clinical guidelines for the management of children with Wilms tumour in a low income setting. Pediatr Blood Cancer 60(1):5-11

13. D'Angio GJ (2003) Pre- or post-operative treatment for Wilms tumor? who, what, when, where, how, why-and which. Med Pediatr Oncol 41 (6):545-549

14. John R, Kurian JJ, Sen S, Gupta MK, Jehangir S, Mathew LG et al (2018) Clinical outcomes of children with Wilms tumor treated on a SIOP WT 2001 protocol in a tertiary care hospital in south India. J Pediatr Urol 14(6):547. e1-547.e7

15. Fawkner-Corbett DW, Howell L, Pizer BL, Dominici C, McDowell HP, Losty PD (2014) Wilms' tumor--lessons and outcomes--a 25-year single center UK experience. Pediatr Hematol Oncol 31(5):400-408

16. Hall G, Grant R, Weitzman S, Maze R, Greenberg M, Gerstle JT (2006) Predictors of surgical outcome in Wilms' tumor: a single-institution comparative experience. J Pediatr Surg 41(5):966-971

17. Wilde JC, Lameris W, van Hasselt EH, Molyneux EM, Heij HA, Borgstein EG (2010) Challenges and outcome of Wilms' tumour management in a resource-constrained setting. Afr J Paediatr Surg 7(3):159-162

18. Pritchard-Jones K, Graf N, van Tinteren H, Craft A (2016) Evidence for a delay in diagnosis of Wilms' tumour in the UK compared with Germany: implications for primary care for children. Arch Dis Child 101(5):417-420

19. Verma N, Kumar A (2016) Clinicoepidemiological profile and outcome of children with Wilms tumor in a developing country. J Pediatr Hematol Oncol 38(7):e213-e216

20. Sultan I, Masarweh M, Ismael T, Al-Hussaini M, Almousa A, Ali HM et al (2009) From upfront nephrectomy to preoperative chemotherapy and back: a single institution experience in the treatment of Wilms tumor. J Pediatr Hematol Oncol 31(5):333-338

21. Israels T, Pidini D, Borgstein E, Bailey S, Tump C, Chagaluka G et al (2018) Survival of children with a Wilms tumor in Blantyre, Malawi. Pediatr Hematol Oncol 35(3):196-202
22. Cafferata C, Cacciavillano W, Galluzzo ML, Flores P, Rose A, Zubizarreta P (2017) Outcome of nephroblastoma treatment according to the SIOP-2001 strategy at a single institution in Argentina. J Pediatr Hematol Oncol 39(1): 50-55

23. Zakaria OM, Hokkam EN, Sayem KA, Daoud MYl, Zakaria HM, Sedky F et al (2015) Initial surgery in tailoring treatment for children with stage II and II Wilms' tumor: an experience from resource challenged settings. World J Oncol 6(5):441-445

24. Elgendy A, Shehata S, Medhat Zaki A, Shehata S (2019) National survey on the management of wilms tumor. J Pediatr Hematol Oncol 41(4):280-285

25. Bhatnagar S (2009) Management of Wilms' tumor: NWTS vs SIOP. J Indian Assoc Pediatr Surg 14(1):6-14

26. Naguib SF, El Haddad A, El Badawy SA, Zaghloul AS (2008) Multidisciplinary approach to Wilms' tumor: a retrospective analytical study of 53 patients. J Egypt Natl Canc Inst 20(4):410-423

27. Godzinski J, Audry G, Cecchetto G, Fuchs J, Irtan S, Okoye B, et al. (2017) The violations of the surgical guidelines for wilms tumour nephrectomy. The $49^{\text {th }}$ Congress meeting of International Society of Paediatric Oncology (SIOP). Pediatr Blood Cancer.64 Suppl 3.

28. Godzinski J, de Kraker J, Graf N, Pritchard-Jones K, Bergeron C, Heij H et al (2004) Is the number of lymph nodes sampled at Wilms' tumour nephrectomy predictive for detection of the regional extention of the disease? Pediatr Blood Cancer 43:303-503

29. Kieran K, Anderson JR, Dome JS, Ehrlich PF, Ritchey ML, Shamberger RC et al (2012) Lymph node involvement in Wilms tumor: results from National Wilms Tumor Studies 4 and 5. J Pediatr Surg 47(4):700-706

30. Gow KW, Barnhart DC, Hamilton TE, Kandel JJ, Chen MK, Ferrer FA et al (2013) Primary nephrectomy and intraoperative tumor spill: report from the Children's Oncology Group (COG) renal tumors committee. J Pediatr Surg 48(1):34-38

\section{Publisher's Note}

Springer Nature remains neutral with regard to jurisdictional claims in published maps and institutional affiliations.

\section{Submit your manuscript to a SpringerOpen ${ }^{\circ}$ journal and benefit from:}

- Convenient online submission

- Rigorous peer review

- Open access: articles freely available online

High visibility within the field

- Retaining the copyright to your article

Submit your next manuscript at $>$ springeropen.com 